\title{
Minimizing the Arrayed Waveguide Grating Cost and the Optical Cable Cost in Deploying WDM Passive Optical Networks
}

\author{
Jingjing Zhang and Nirwan Ansari
}

\begin{abstract}
We investigate the issue of minimizing the cost of optical cables and arrayed waveguide gratings (AWGs) in deploying optical distribution networks using WDM passive optical networks (PONs). Generally, when deploying WDM PONs with cascaded AWGs, increasing the number of stages of cascaded AWGs decreases the optical fiber costs, but increases the AWG cost. A proper cascaded AWG structure and proper connections between AWGs and optical network units are needed to minimize the total cost of AWGs and optical cables. We decompose the network planning problem into two subproblems. One is to decide the positions of AWGs and placement of optical cables to minimize the cost of optical cables. The other one is to determine the cascaded AWG structure to minimize the total cost of AWGs and optical cables. In particular, we propose a recursive partitioncombination-based algorithm to achieve the optimal trade-off between the AWG cost and the optical cable cost.
\end{abstract}

Index Terms-Network planning; WDM PON; AWG.

\section{INTRODUCTION}

$\mathbf{T}$ he WDM passive optical network (PON) is a future-proof broadband access technology that exploits the high bandwidth of optical fibers [1-3]. It generally employs arrayed waveguide gratings (AWGs) to demultiplex fibers into multiple wavelengths, each of which is assigned to one or more subscribers. Owing to their cyclic property, AWGs can be cascaded to realize the demultiplexing function $[4,5]$. To realize the same demultiplexing function, there are

Manuscript received April 16, 2009; revised July 13, 2009; accepted July 23, 2009; published September 4, 2009 (Doc. ID 109876).

J. Zhang (e-mail: jz58@njit.edu) and N. Ansari (e-mail: nirwan.ansari@njit.edu) are with the Advanced Networking Laboratory, Department of Electrical and Computer Engineering, New Jersey Institute of Technology, Newark, New Jersey 07032, USA.

Digital Object Identifier 10.1364/JOCN.1.000352 many options in designing cascaded AWG structures. These cascaded AWG structures use different sizes and numbers of AWGs, hence yielding different AWG costs [6-9]. In addition, different cascaded AWG structures are associated with different optical cable placements and may introduce different costs of optical fiber cables as well. Generally, increasing the number of stages of cascaded AWGs used in an optical distribution network (ODN) decreases the optical cable cost but increases the AWG cost. Minimizing the total cost of cascaded AWGs and optical fibers is an important issue for catalyzing the deployment of WDM PON, especially in deploying long-reach PONs with a broad coverage area with diversified subscriber density [10].

Let us first consider the following example as shown in Fig. 1(a). The optical line terminal (OLT) is located at the center of a circle. Four optical network units (ONUs) are uniformly distributed on the circumference of the circle. We have two schemes of placing AWGs and optical cables to deliver one wavelength to each ONU. The first scheme, as shown in Fig. 1(b), is to use one $1 \times 4$ AWG. This AWG is placed at the center of the circle and connected to four ONUs by four individual optical fibers. The total length of fiber is $4 r$, where $r$ is the radius of the circle. An alternative scheme is to use cascaded AWGs with three AWGs of size $1 \times 2$, as shown in Fig. 1(c). Among the three AWGs, one is placed at the center of the circle, and the other two are placed between ONUs and the OLT. The total length of the optical cable is $(\sqrt{2}+\sqrt{6}) r$, which is less than $4 r$. As compared with the first scheme, the second scheme requires shorter optical cables and AWGs of smaller sizes. However, the number of AWGs is increased from one to three. The AWG cost of the second scheme is probably greater than that of the first scheme. Which scheme will yield the minimum total cost depends on the radius $r$ (determined by subscriber density), optical cable cost, cable deployment cost, and the cost of AWGs of different sizes. Generally, there exists a trade-off between the AWG cost and the optical cable cost. 


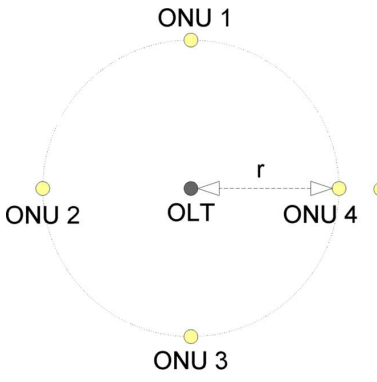

(a)

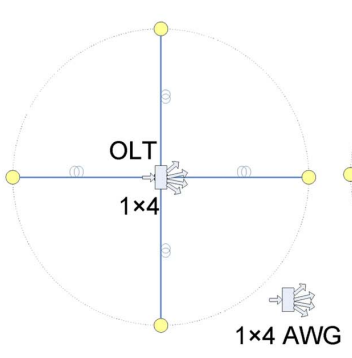

(b)

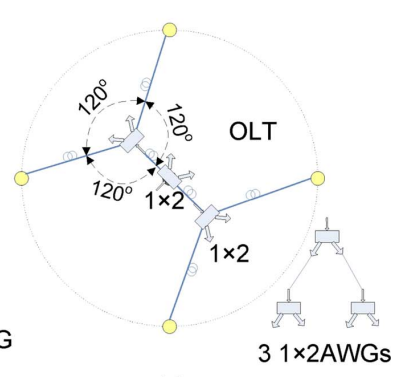

(c)

Fig. 1. (Color online) Simple example of the AWG cost and the optical cable cost.

The problem of planning an ODN using a WDM PON can be generally described as follows: given the geometric positions of the OLT and ONUs as well as the price of AWGs and optical fiber cables, minimize the total cost of AWGs and optical fiber cables by selecting AWGs of proper sizes, finding suitable locations for AWGs, and placing optical cables properly. Formerly, many works concentrated on planning PONs with the minimum optical cable cost [11-13]. Without considering the AWG cost, this problem is reducible to the Euclidean Steiner tree problem, which is proved NP-hard [14]. Besides the link cost considered in the Steiner tree problem, the node cost of AWGs has to be taken into consideration as well in solving the optimization problem. In this paper, we concentrate on optimizing the trade-off between the AWG cost and optical fiber cable cost instead of focusing on solving the Steiner tree problem. In particular, we consider the network planning problem in the scenario in which existing constructions can be utilized. Since most of the areas were deployed with constructions for other service infrastructures, e.g., cable TV service, utilizing these existing constructions can save the expensive conduit deployment cost $[11,15,16]$. We assume that the existing construction forms a tree structure with the OLT as the root, and subscribers are distributed along the tree.

In this paper, we employ a divide-and-conquer strategy to optimize the trade-off between the AWG cost and the optical cable cost. Generally, the problem can be divided into two subproblems. First, given a cascaded AWG structure, minimize the optical fiber cable cost by properly placing AWGs and optical cables; this is referred to as Problem 1, which can be further divided into two subproblems: determine the subscribers connected to each AWG, and decide geometric locations of AWGs. The two subproblems are referred to as Problems 1.1 and 1.2, respectively, where Problem 1.1 is NP-hard. After solving Problem 1 , each cascaded AWG structure will be associated with a minimum optical cable cost. The remaining problem is to determine the cascaded AWG structure with the minimum total cost of AWGs and optical cables; this is referred to as Problem 2. For the AWG cost, the most economic scheme is to use one big-size AWG for all subscribers. However, this incurs a high optical cable cost, since each subscriber needs at least one individual optical fiber cable to connect to the AWG. The alternatives are using multistage cascaded AWGs of smaller sizes. In this case, the optical cable cost is reduced at the sacrifice of increasing the AWG cost, as shown in the example discussed before. To determine the best trade-off, one scheme is to list all cascaded AWG structures and then calculate the total incurred costs for each of them. However, this scheme is time consuming, since the number of combinations increases exponentially with the increase of subscribers. An efficient algorithm is greatly needed to solve Problem 2.

The contributions of this paper include the following: a) we propose to apply heuristic tree-partitioning algorithms to determine ONUs connected to each AWG in solving Problem 1.1; b) for Problem 1.2, we place AWGs into proper locations in the tree to minimize the cost of optical fiber cables; c) we propose a recursive partition-combination algorithm to avoid the exhaustive search of every combination of AWGs in addressing Problem 2. We assume that each ONU uses one wavelength for upstream and one for downstream data transmission. The upstream and downstream wavelengths of an ONU will be routed to the same output port of an AWG and be delivered in a single bidirectional fiber. The upstream signal and downstream signals are separated by a circulator or a wavelength filter at the ONU side. The proposed scheme in this paper can be easily tailored to the case with each subscriber using any number of wavelengths and any number of optical fibers. It can also be applied to the planning of TDM PONs and hybrid WDM/TDM PONs.

The rest of the paper is organized as follows. In Section II, we discuss the AWG device as well as the cascaded AWG structure. Section III presents the problem formulation. Section IV covers our proposed algorithms in detail. Section V presents the analysis of our proposed algorithms. Concluding remarks are given in Section VI. 


\section{CASCADED AWGS}

In this section, we discuss cascaded AWG structures as well as their costs in detail.

\section{A. Cascaded AWGs}

An AWG is used to realize the demultiplexing function in the WDM PON [4]. Figure 2(a) illustrates the prototype of a typical $N \times N$ AWG. Let $N$ be the number of AWG ports, $W$ be the number of wavelengths in one fiber, and $\lambda_{1}, \lambda_{2}, \ldots, \lambda_{W}$ be the working wavelengths of the AWG device. These wavelengths are equally spaced. Signals in the AWG are cyclically routed with the rule that wavelength $\lambda_{f}$ from input $i$ is routed to output $\bmod (i-2+f, N)+1$.

Owing to the cyclical property of AWGs, multiple wavelengths can be routed to a single fiber, thus facilitating AWG cascading. Maier et al. [6] analyzed the cascaded AWG structure in detail. Figure 2(b) shows a general cascaded AWG structure.

Let vectors (in, out) describe the demultiplexing capability of any cascaded AWG structure, where in is the number of input ports of AWGs in the first stage, and out is the number of output ports of AWGs in the last stage. In terms of PON, let $F$ be the number of optical fibers from the OLT, $N$ be the number of subscribers, and $W$ be the number of wavelengths in the fiber at the OLT. Then, for the cascaded AWG employed in the PON, the number of input ports in equals $F$, and the number of output ports out should be no less than $N$. Since each ONU needs two wavelengths, one for upstream and one for downstream transmission, the total number of wavelengths of all optical fibers at the OLT $F \times W$ should be no less than $2 N$, i.e., $F \times W \geqslant 2 N$. Let $K$ be the number of stages, $n_{k}$ be the number of AWGs in stage $k$, and $i n_{k}$ and out $t_{k}$ be the number of used input ports and output ports of AWGs in stage $k$, respectively. Then,

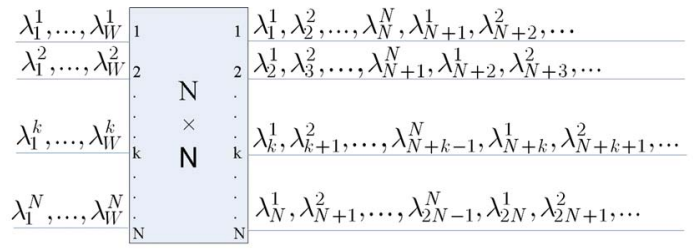

(a)

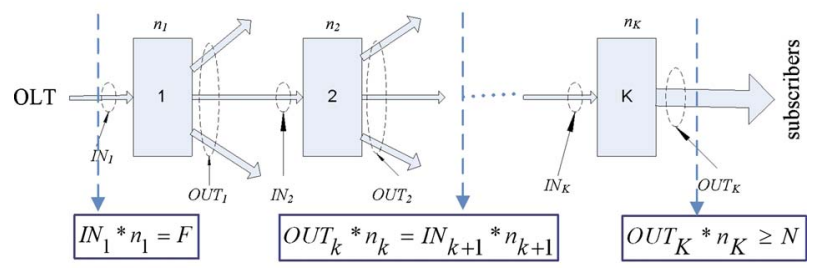

(b)

Fig. 2. (Color online) Abstracted functionality of a typical AWG.
- for the first stage 1 ,

$$
i n_{1} \times n_{1}=F
$$

- for any intermediate stage $k$,

$$
\text { out }_{k} \times n_{k}=i n_{k+1} \times n_{k+1} \text {; }
$$

- for the last stage $K$,

$$
\text { out }_{K} \times n_{K} \geqslant N \text {, where } 2 N \leqslant F \times W \text {. }
$$

In this paper, we assume that the AWGs support 2, $4,8,16,32$, and 64 ports, which are the most popular sizes commercially available. We specifically use equally spaced input ports so that channels in each output port of these AWGs are equally spaced, thus facilitating further cascading.

To realize the same demultiplexing functionality, different AWG combinations can be employed. Figure 3 shows two examples of equal-function cascaded AWGs with different costs. The structure as shown in Fig. 3(a) uses one AWG with size $2 \times 4$, whereas the structure as shown in Fig. 3(b) uses two AWGs, each of which is of size $1 \times 2$. Both of these two architectures demultiplex wavelengths from two fibers into four fibers. In Figs. 3(c) and 3(d), wavelengths in one fiber are demultiplexed into four fibers. The structure as shown in Fig. 3(c) uses one AWG with size $1 \times 4$, whereas the structure as shown in Fig. 3(d) uses twostage cascaded AWGs, where the AWG with size $1 \times 2$ in the first stage is connected to two AWGs with size $1 \times 2$ in the second stage. For the two cascaded AWG structures with the same demultiplexing functionality, besides the differences in the number and sizes of AWGs, they may also differ in the coarseness of employed AWGs. Coarseness, defined as the number of contiguous wavelength channels routable on the same output ports, characterizes the optical resolution of the AWG [6]. AWGs in Fig. 3(a) and 3(b) have the same coarseness with one wavelength channel, and AWGs in Fig. 3(c) and 3(d) can have different coarseness. The coarseness of the AWG in Fig. 3(c) is with one wavelength, whereas, in Fig. 3(d), the coarseness

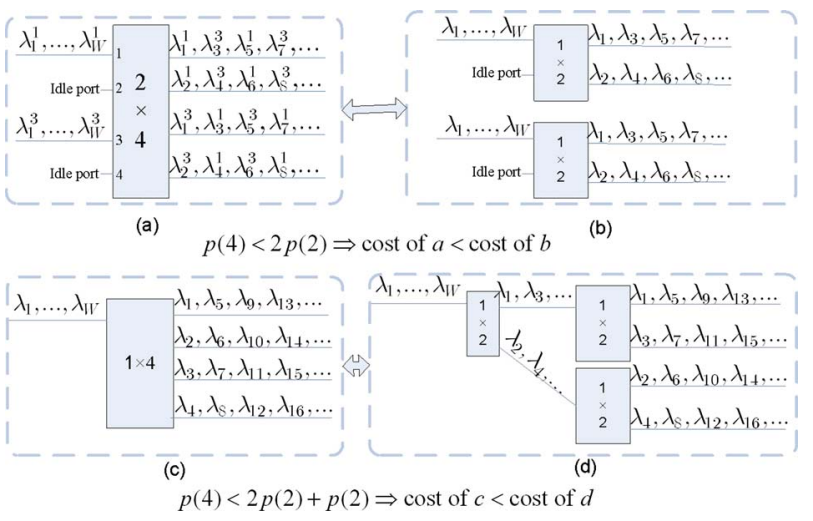

Fig. 3. (Color online) Equal-function cascaded AWGs. 
of the AWGs in the second stage can be increased to two contiguous wavelength channels for lower resolution.

\section{B. Cost of Cascaded AWGs}

Different AWG combinations can realize the same demultiplexing function. However, they introduce different costs. In this paper, we assume the price of an AWG is a function of the number of its output ports. Let $p(x)$ be the price of AWG with $x$ ports; $p(x)$ possesses the following properties:

- The larger the number of AWG ports, the higher the price of the AWG, i.e.,

$$
p(x)>p(y), \quad \forall x>y .
$$

- The larger the number of AWG ports, the smaller the price per port, i.e.,

$$
p(x) / x<p(y) / y, \quad \forall x>y .
$$

For the structures shown in Fig. 3, the one in Fig. $3(\mathrm{a})$ costs $p(4)$, the one in Fig. $3(\mathrm{~b})$ costs $2 \times p(2)$, the one in Fig. 3(c) costs $p(4)$, and the one in Fig. 3(d) costs $3 \times p(2)$. Based on these two assumptions, AWGs in Figs. 3(a) and 3(c) cost less than those in Figs. 3(b) and $3(\mathrm{~d})$, respectively.

For the cascaded AWGs employed in the PON, $n_{K}$ $\times p\left(\right.$ out $\left._{K}\right)$ is the AWG cost in the last stage; $i n_{K} \times n_{K}$ $\times p\left(\right.$ out $\left._{K-1}\right) /$ out $_{K-1}$ is the AWG cost in the stage before the last; and by recursion, the AWG cost in stage $k$ is $n_{K} \times$ out $_{K} \times\left(\Pi_{k+1}^{K}\right.$ in $_{i} /$ out $\left._{i}\right) \times p\left(\right.$ out $\left._{k}\right) /$ out $_{k}$. Then, the total AWG cost is

$$
n_{K} \times \text { out }_{K} \times \sum_{k=1}^{K}\left(\prod_{k+1}^{K} \frac{\text { in }_{i}}{\text { out }_{i}} \times \frac{p\left(\text { out }_{k}\right)}{\text { out }_{k}}\right) .
$$

Based on the assumption of prices as shown in Eqs. (4) and (5), as well as the constraints of the cascaded AWG structure as shown in Eqs. (1)-(3), minimizing Eq. (6) requires that the number of output ports of the AWGs, out ${ }_{k}$, should be as large as possible. From the assumption of the AWG port number, we further derive the following regarding the AWG cost in cascaded AWGs:

- To demultiplex in fibers into out ONUs, the cost of cascaded AWGs is minimized by using one large AWG of size in $\times$ out.

- To demultiplex one fiber into out ONUs, the secondary choice is a two-stage cascaded AWG consisting of two AWGs of size $1 \times$ out $/ 2$ plus one AWG of size $1 \times 2$.

- To demultiplex in (in $\geqslant 2$ ) fibers into out ONUs, the secondary choice is using two AWGs with size in $/ 2 \times$ out $/ 2$.

These properties will be incorporated into our proposed algorithm.

\section{PROBLEM FORMULATION}

In this section, we describe and mathematically formulate the problem of planning the ODN to minimize the cost of AWGs and optical cables. The general problem of ODN planning can be formulated as follows:

Given the number of optical cables at the OLT, the wavelengths to be demultiplexed, the number of ONUs as well as their geometric locations, the number of wavelengths required by each ONU, the price of AWGs, and the price of optical cables,

Obtain the cascaded AWG architecture, the positions of AWGs, and the placement of optical cables.

Objective: minimize the total cost of AWGs and optical cables.

Without considering the AWG cost, the problem of minimizing the total length of links connecting the OLT and ONUs in greenfield planning is reduced to the Euclidean Steiner tree problem, which is proved to be NP-hard [14]. In this paper, we do not focus on solving the Steiner tree problem. Since most of the areas have been deployed with constructions for other service infrastructures, e.g., cable television [16], we utilize these existing constructions and do not consider the expensive deployment cost. We assume that the existing construction forms a tree structure with the OLT as the root. ONUs are distributed along the edges of the tree. This tree is referred to as a "construction tree" in this paper. Our focus is to place AWGs and optical cables over the construction tree to optimize the trade-off between the AWG cost and the optical cable cost. The greenfield planning will be addressed in our future work.

Given the tree topology, the problem is reduced to deciding the cascaded AWG structure as well as the positions of AWGs and the placement of optical cables. This problem can be mathematically formulated as follows:

\section{Given}

1) $T$, the construction tree formed by the existing constructions;

2) $N$, the number of ONUs;

3) $F$, the number of optical cables at the OLT;

4) $W$, the number of wavelengths in optical cables (where $F \times W$ should be no less than $2 N$ to guarantee enough wavelengths be supplied to ONUs);

5) The geometric positions of ONUs;

6) $p(x)$, the price of the AWG with $x$ ports, where $x$ equals $2,4,8,16, \ldots$;

7) $q(x)$, the unit price of optical cables containing $x$ optical fibers;

Obtain

1) $K$, the number of stages of cascaded AWGs; 
2) $i n_{k}$, the number of input ports of AWGs at stage $k$

3) out $_{k}$, the number of output ports of AWGs at stage $k$;

4) The positions of each AWG;

5) The connections between AWGs and ONUs, connections among AWGs, and connections between AWGs and OLT.

Objective:

1) $\operatorname{minimize}\left(\Sigma \mathrm{AWG}\right.$ cost $+\sum$ optical cable cost $)$

Subject to

1) Each ONU is assigned with two wavelengths.

The solution of this problem is presented next.

\section{Minimize the Total Cost of AWGs AND Optical CABLES}

In this section, we discuss strategies to minimize the total cost of AWGs and optical fiber cables. We decompose the original problem into two subproblems. First, given a cascaded AWG structure, we place AWGs into proper positions and connect them to proper ONUs to minimize the optical cable cost. Then, each cascaded AWG structure is associated with an optical cable cost as well as an AWG cost. The second problem is to derive the cascaded AWG structure that yields the minimum total cost of AWGs and optical cables. The former problem is referred to as Problem 1 , whereas the latter problem is referred to as Problem 2. The solution of Problem 1 determines the solution of Problem 2. Figure 4(a) shows the decomposition of the original problem. We next discuss these two problems in detail.

\section{A. Minimize the Optical Cable Cost for a Given Cascaded AWG (Problem 1)}

Given the positions of the OLT and subscribers, as well as the cascaded AWG structure, AWGs need to be

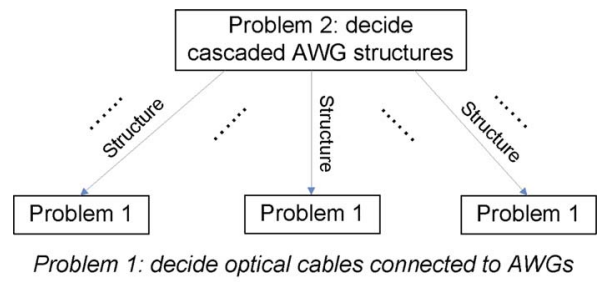

(a) The decomposition of the original problem

\begin{tabular}{|c|}
\hline $\begin{array}{c}\text { Problem 1.1: decide ONUs } \\
\text { connected to AWGs }\end{array}$ \\
\hline $\begin{array}{c}\text { Problem 1.2: decide the } \\
\text { positions for AWGs }\end{array}$ \\
\hline
\end{tabular}

(b) The decomposition of problem 1

Fig. 4. (Color online) Decomposition of the problem. connected with proper ONUs and placed at proper positions to minimize the optical cable cost.

We refer to the AWG connected directly to subscribers as the "distribution AWG," otherwise as the "intermediate AWG." ONUs are connected to distribution AWGs by optical cables. Distribution AWGs are connected to intermediate AWGs or the OLT by optical cables. These deployed optical cables form a tree structure with the OLT as the root and ONUs as the leaves. This tree is referred to as the "optical cable tree." Note that the optical cable tree is built on the construction tree. The vertex set of the optical cable tree is a subset of the vertex set of the construction tree. The edge connecting $(u, v)$ in the optical cable tree is actually the route connecting vertex $u$ and vertex $v$ in the construction tree. Figures 5(a) and 5(b) illustrate an example of the construction tree and the optical cable tree, respectively. In Fig. 5(a), 16 ONUs are distributed along the construction tree. In Fig. 5(b), a two-stage cascaded AWG structure is used to demultiplex wavelengths to 16 ONUs. The AWG in the first stage as well as those four AWGs in the second stage are all of size $1 \times 4$. The AWG in the first stage is connected to AWGs in the second stage by optical cables. AWGs in the second stage are connected to ONUs by optical cables.

Each AWG is connected to some ONUs or AWGs by optical cables. Figure 5(c) shows five subtrees corresponding to five AWGs and their respective children as shown in Fig. 5(b). The total optical cable cost is the sum of the optical cable cost in all subtrees. Given an AWG, the cost of optical cables connecting to this particular AWG with its children can be derived as fol-

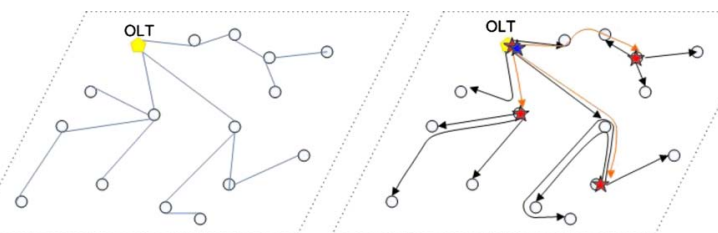

(a) The construction tree

(b) The optical cable tree $\bigcirc$ ONU $\star$ AWG in stage 1

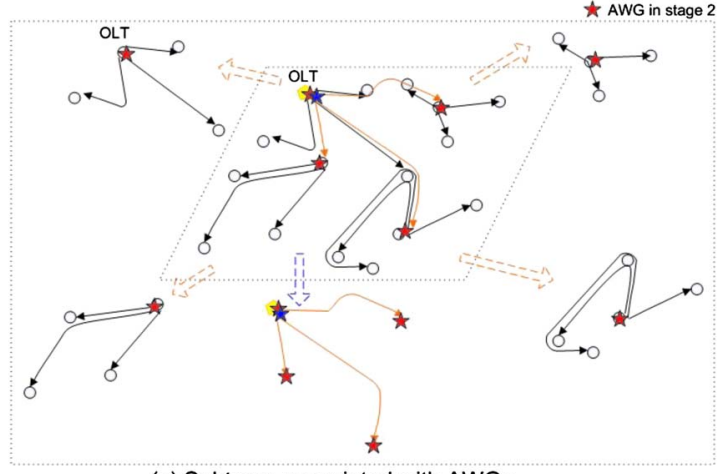

(c) Subtrees associated with AWGs

Fig. 5. (Color online) Example of construction tree, optical cable tree, and subtrees associated with AWGs. 
lows. Let the AWG be designated as the root of the subtree formed by the AWG and its children. The following can be derived for the optical cable cost.

Assume $u$ and $v$ are two vertices of the subtree. Let $|(u, v)|$ be the length of edge $(u, v)$, and $w_{(u, v)}$ be the weight of edge $(u, v)$. Without loss of generality, assume $u$ is the parent node of $v \cdot w_{(u, v)}$ is defined as the number of child nodes of node $v$. It implies that $w_{(u, v)}$ optical fibers have to be placed on edge $(u, v)$ for $w_{(u, v)}$ children. Each of the fibers is a bidirectional fiber delivering both upstream and downstream signals. Then, the cost of optical cables in the subtree $t$ for this particular AWG is

$$
\sum_{(u, v) \in E(t)} q\left(w_{(u, v)}\right)|(u, v)|
$$

Here, $E(t)$ denotes the set of edges of tree $t$. Figure 6(a) shows one example of weights of edges in a subtree. Assume all edges are of equal length of 1 ; the total optical cable cost is $5 q(1)+q(2)+q(3)$.

Equation (7) describes the cost of optical cables in the subtree constituted by one AWG and its children. Each AWG is associated with a subtree constituted by the AWG itself as well as its children. Let $m$ be the number of AWGs. The total optical cable cost is

$$
\sum_{i=1}^{m} \sum_{(u, v) \in E\left(s t_{i}\right)} q\left(w_{(u, v)}\right)|(u, v)|
$$

Then, the problem of minimizing the optical cable cost is equivalent to minimizing Eq. (8). If $q(x)$ $=1 \forall x$, the problem is reduced to the treepartitioning problem [17], which is NP-complete. So, the problem with any $q(x)$ is NP-complete.

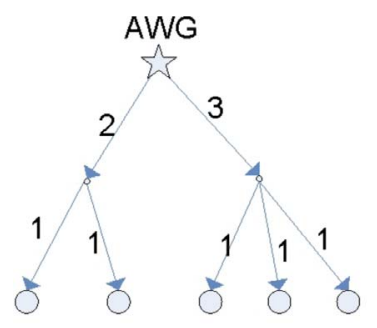

(a) An example of $W_{(u, v)}$

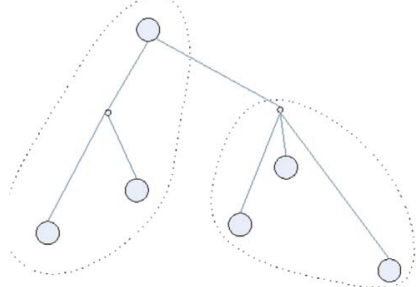

(b) An example of tree partition

Fig. 6. (Color online) Example of weights of edges and an example of tree partitioning.
To solve this problem, we decompose it into two subproblems: determine children connected to each AWG, and decide the geometric position for each AWG. The two problems are referred to as Problems 1.1 and 1.2, respectively. Figure 4(b) shows the decomposition of Problem 1:

In the following, we discuss Problems 1.1 and 1.2 in detail.

1) Tree Partition (Problem 1.1). Specifically, Problem 1.1 is to partition the construction tree into subtrees with the objective of minimizing the total link length of the optical cable tree:

$$
\sum_{i=1}^{m} \sum_{(u, v) \in E\left(s t_{i}\right)}|(u, v)| .
$$

As compared with Problem 1 of minimizing Eq. (7), Problem 1.1 disregards the weight of each edge.

Since the number of ONUs is usually much greater than the number of AWGs, the cost of optical cables for connections between ONUs and distribution AWGs far exceeds that for connections between AWGs. In solving Problem 1.1, we focus on minimizing the total optical cable cost of the subtrees associated with distribution AWGs, but disregard that of subtrees associated with intermediate AWGs. The problem of partitioning construction tree $T$ into subtrees associated with distribution AWGs is formulated as follows:

Given an ONU set $V$ and the construction tree $T$,

Obtain a partition $V_{1}, V_{2}, \ldots, V_{m}$, where $m$ is the number of distribution AWGs, $V_{i} \cap V_{j}=\varnothing, \forall i \neq j$.

Objective: $\operatorname{minimize}\left(\Sigma_{i} \Sigma_{(u, v) \in E\left(s t_{i}\right)}|(u, v)|\right)$, where $s t_{i}$ is the subtree formed by $V_{i} \forall i$.

This tree-partitioning problem is proved NPcomplete [17]. Many proposed heuristic algorithms, such as the bottom-up algorithm [18], can be employed to solve this problem. Figure 6(b) shows one example of tree partitioning.

2) AWG placement (Problem 1.2). The above discussed solution of Problem 1.1 determines leaves of each subtree. Problem 1.2 is to decide geometric positions for the root of each subtree. Actually, Problem 1.2 is equivalent to the minimization problem with the following objective:

$$
\sum_{(u, v) \in E\left(s t_{i}\right)} q\left(w_{(u, v)}\right)|(u, v)| \quad \forall i .
$$

As compared with Problem 1 of minimizing Eq. (7), Problem 1.2 focuses on minimizing the optical cable cost of one subtree only. We begin from minimizing the cost of optical cables connecting ONUs and distribution AWGs.

The optical cable cost of a particular subtree, as described in Eq. (10), depends on the lengths and 
weights of its edges. Since the lengths of edges in the subtree are determined by solving Problem 1.1, we want to adjust the weights of edges so as to minimize the optical cable cost of the subtree. The weights of edges will be different when different vertices are designated as the root of the subtree, where the AWG is placed. The problem is equivalent to assigning a proper vertex as the root of a subtree to minimize the optical cable cost. Our method is to designate the centroid of a subtree as its root, which is defined as follows.

Definition 1. Given a tree $T=(V, E)$ with $n$ vertices, let $s t_{1}, s t_{2}, \ldots, s t_{m}$ be the generated subtrees after removing vertex $v$. Vertex $v$ is referred to as the centroid of the tree if $\left|s t_{i}\right| \leqslant n / 2, \forall i$.

In Fig. 7(a), both vertex $c$ and vertex $d$ are centroids of the tree. In Figs. 7(b) and 7(c), vertex $d$ is the centroid of the tree.

The centroid of the tree possesses the following property.

Property 1. Given the tree consisting of ONUs to be connected to one AWG, the centroid of the tree is the optimal position for the AWG to yield the minimum optical cable cost.

Proof: For any two vertices, $u, v,(u, v) \in E$, let the size of subtrees generated by removing $u$ be $s t_{1}, s t_{2}, \ldots, s t_{m}$. Without loss of generality, assume that $v$ is in subtree $s t_{1}$. If the AWG is placed at $u$, the number of fibers in $(u, v)$ equals $\left|s t_{1}\right|$; if the AWG is placed at $v$, the number of fibers in $(u, v)$ equals $\sum_{i=2}^{m}\left|s t_{i}\right|$; for the above two placement schemes, the fiber placements are the same in all the other edges except edge $(u, v)$. So, $v$ is preferred over $u$ for a lower optical cable cost if and only if $\sum_{i=2}^{m}\left|s t_{i}\right|<\left|s t_{1}\right|$. In other words, when there is no subtree $i$ satisfying $\left|s t_{i}\right|>\Sigma_{j \neq i}\left|s t_{j}\right|$, the corresponding vertex is the optimal position of AWG, which is the centroid of the tree.

In Fig. 7(b), the AWG is placed into vertex $c$. The optical cable in edge $(c, d)$ has to contain three optical fibers. In Fig. 7(c), the AWG is placed into vertex $d$. The optical cable in edge $(c, d)$ contains two optical fibers.

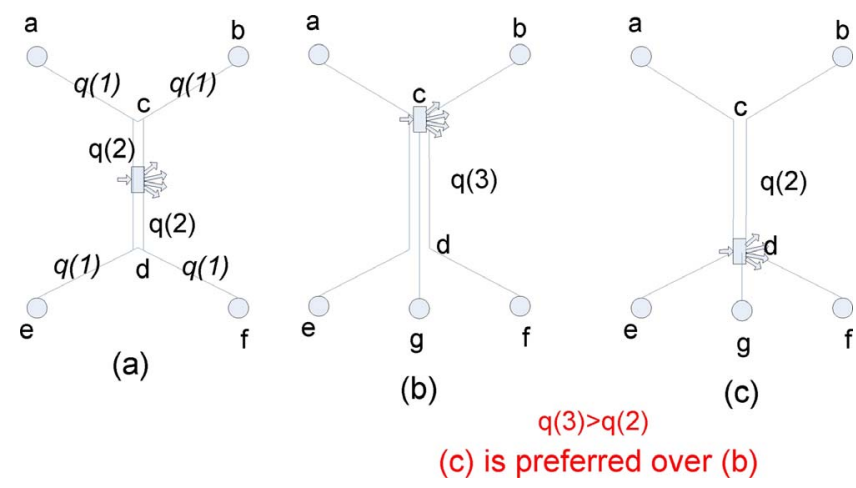

Fig. 7. (Color online) Centroid of trees.
Hence, vertex $c$ is preferred over vertex $d$ for the AWG position. Actually, vertex $d$ is the centroid of the tree, which is the optimal position for the AWG.

Hence, for the network as shown in Fig. 7(a), the AWG can be placed at any point between vertex $c$ and vertex $d$. For the network as shown in Fig. 7(b) and 7 (c), the AWG can be placed at vertex $d$.

By using the same method, the positions of intermediate AWGs can be decided as well.

By solving Problems 1.1 and 1.2, we have therefore solved Problem 1 of minimizing the optical cable cost for a given cascaded AWG structure. We continue to solve Problem 2 of deciding the cascaded AWG structure with the minimum total cost.

\section{B. Decide the Cascaded AWG Structure With the Minimum Total Cost (Problem 2)}

Each cascaded AWG structure is associated with two kinds of costs. One is the AWG cost. The other one is the minimum optical cable cost connecting the OLT, AWGs, and ONUs, which is the objective of Problem 1. To derive the cascaded AWG structure with the minimum total cost of AWGs and optical cables, one way is to calculate the total cost of every possible AWG combination and then select the right one. However, the number of AWG combinations possesses the following property.

Property 2. Let $\mathcal{N}(x)$ be the number of $A W G$ combinations that demultiplex one fiber into $x$ ONUs; i.e., the demultiplexing capability has to be $(1, x)$. Then, $\mathcal{N}\left(2^{k}\right)=\left[\mathcal{N}\left(2^{(k-1)}\right)\right]^{1^{1}}+\left[\mathcal{N}\left(2^{(k-2)}\right)\right]^{2^{2}}+\ldots+[\mathcal{N}(1)]^{2^{k}}$.

Proof: If the AWG in the first stage is of size $1 \times 2^{i}$, the demultiplexing capability of the remaining stages connecting to each of the $2^{i}$ output ports has to be $\left(1,2^{k-i}\right)$. The number of combinations for this particular $i$ is $\left[\mathcal{N}\left(2^{(k-i)}\right)\right]^{2^{i}} ; i$ ranges from 1 to $k$. Hence, the property is proved.

As we can see, the number of combinations increases exponentially with the increase of subscribers. We have to minimize the total cost of AWGs and optical cables over all cascaded AWG structures to solve Problem 2. However, the minimum optical cable cost associated with one cascaded AWG structure is determined by the solution of Problem 1. Problem 1 further involves Problem 1.1, which is an NP-hard problem. Hence, the total cost cannot be expressed as an explicit function of given AWGs and network parameters. It is difficult to apply existing integer programming methods such as Lagrangian relaxation to obtain the optimal solution. To solve Problem 2, one way is to exhaustively search every combination of AWGs, which requires high computational effort. Instead, we propose a heuristic algorithm referred to as recursive partitioning-combination to acquire a near- 
optimal solution. Recursive partitioning-combination first determines the sizes of distribution AWGs by recursively decreasing the sizes of distribution AWGs and recursively partitioning the tree accordingly. Then, recursive partitioning-combination decides the sizes and stages of intermediate AWGs by recursively increasing the sizes of intermediate AWGs and combining the trees accordingly.

1) Decide sizes of distribution AWGs. Regarding the optical cable cost, Eq. (7) states that a small cost requires small weights of edges; this implies a small size of the subtree. Hence, decreasing the sizes of the subtrees reduces the optical cable cost. In terms of the optical cable cost, distribution AWGs of small sizes are preferred over those of large sizes. However, the quantity of AWGs will be large if AWGs of small sizes are used, thus implying a high cost of AWGs. Which sizes of AWGs will yield the minimum total cost depends on the price of AWGs, optical cables, and subscriber density, as illustrated by the example shown in Fig. 1. We recursively partition the construction tree $T$ and decrease sizes of AWGs to reach the optimal solution.

For any given tree, we consider the cascaded AWG structure (A) with the minimum cost and the cascaded AWG structure (B) with the secondary minimum cost. We can calculate the minimum required optical cable costs for (A) and (B), respectively. If (B) yields a smaller total cost than (A), check the subtrees corresponding to distribution AWGs in (B); this procedure is repeated until the cost of $(B)$ exceeds that of $(\mathrm{A})$. We define two kinds of partition: horizontal and vertical. In the horizontal partition, one large AWG with $x \times y$ is replaced by two parallel AWGs with smaller sizes of $x / 2 \times y / 2$, e.g., from Fig. 3(a) to Fig. 3(b). In the vertical partition, one AWG with size $1 \times y$ is replaced by a

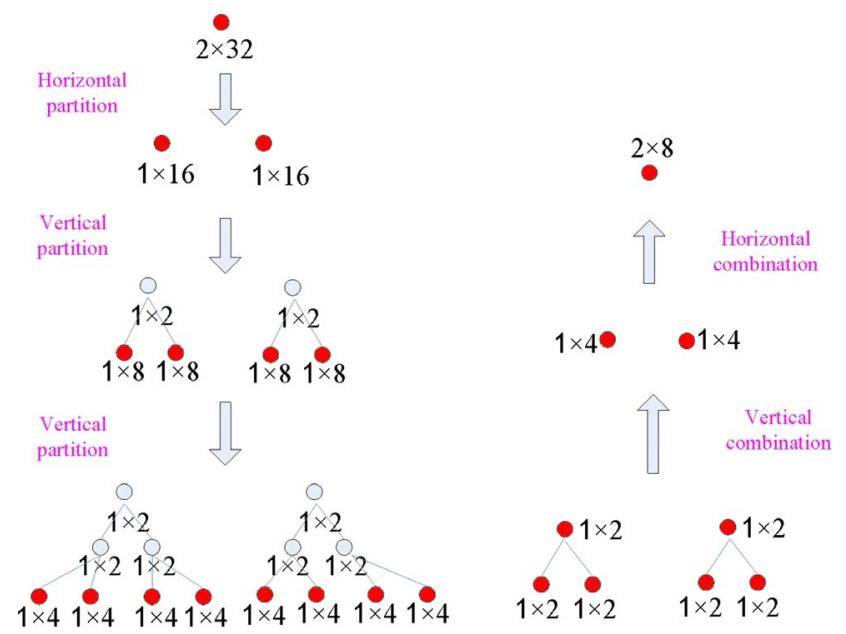

(a) Recursive partition

(b) Recursive combination

Fig. 8. (Color online) Recursive partitioning and recursive combination. two-stage cascaded AWG with smaller sizes of $1 \times 2$ and $1 \times y / 2$, e.g., from Fig. 3(c) to Fig. 3(d). Algorithm 1 describes the procedure in detail. Figure 8(a) shows one example of the recursive partitioning process. In the horizontal partition, an AWG with the size of 2 $\times 32$ is decomposed into two AWGs with the size of 1 $\times 16$. In the vertical partition, one AWG with the size of $1 \times 16$ is decomposed into three AWGs with the sizes of $1 \times 2,1 \times 8$, and $1 \times 8$.

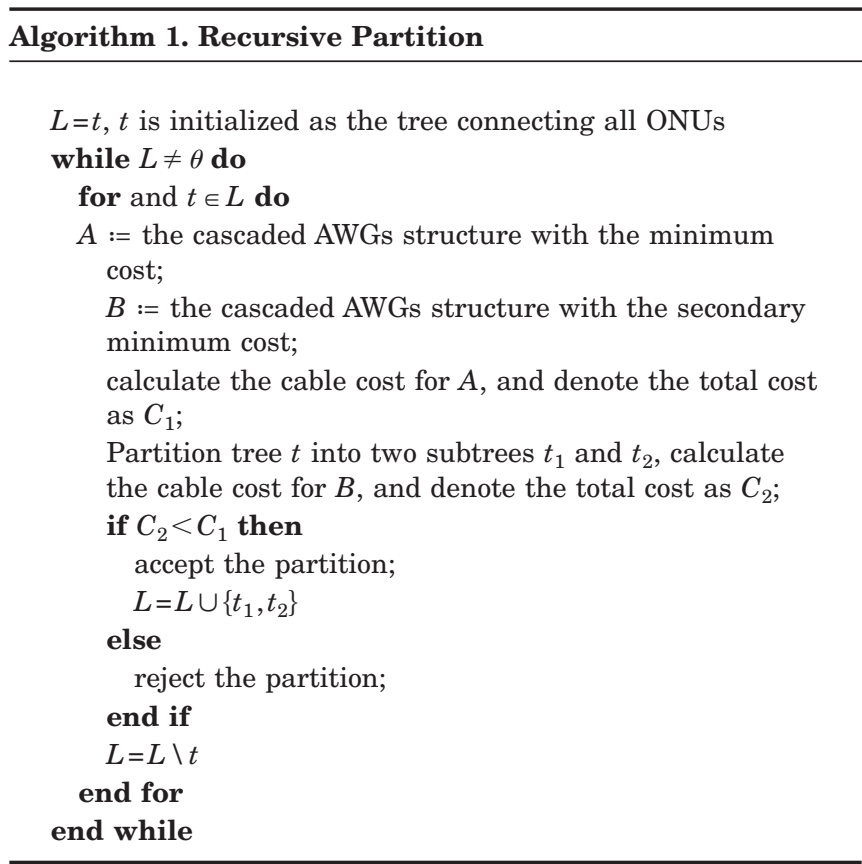

2) Decide sizes and stages of intermediate AWGs. After the above recursive partitioning process, distribution AWGs are connected to OLT via intermediate AWGs with the size $1 \times 2$. We then optimize the sizes and stages of intermediate AWGs by recursive combination. Specifically, these $1 \times 2 \mathrm{AWG}$ are combined into AWGs with bigger sizes from the last stage to the first stage. Similar to the partitioning process, two types of combinations are defined: horizontal combination and vertical combination. The horizontal and vertical combinations are the reverse processes of the horizontal and vertical partition, respectively. Algorithm 2 describes the recursive combination process.

Figure 8(b) shows one example of the recursive combination process. In the vertical combination, twostage cascaded AWGs of size $1 \times 2$ are combined into one-stage AWGs of size $1 \times 4$. In the horizontal combination, two AWGs of size $1 \times 4$ are combined into one AWG of size $2 \times 8$.

We have solved Problems 1 and 2 in the above. We will next analyze the factors affecting the recursive partitioning and combination process. 


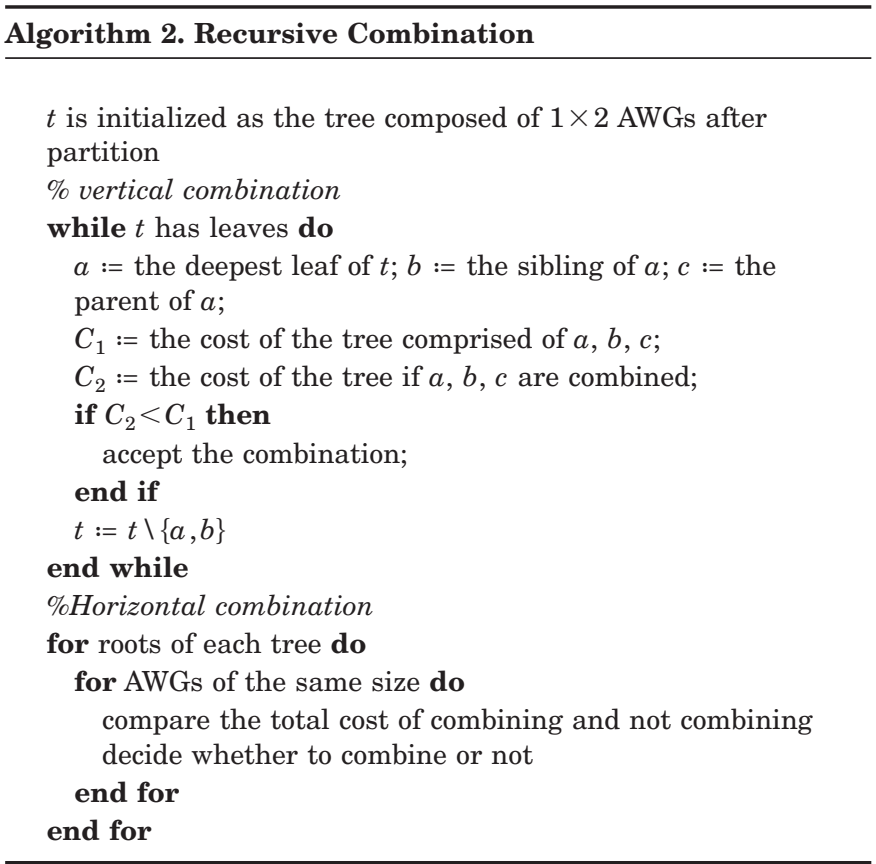

\section{ANALYSIS AND SimUlatiON}

In the above, we employ the divide and conquer strategy to decompose the problem of minimizing the trade-off between the optical fiber cost and the AWG cost into Problems 1 and 2. Problem 1 is further decomposed into Problems 1.1 and 1.2. Problem 1.1 is an NP-hard problem. Problem 1.2 is an easy problem, which we have solved successfully in Subsection IV.A. Problem 2 depends on the solution of Problem 1. To avoid using the exhaustive search, we have employed recursive partitioning and combination to solve Problem 2. The solution to Problem 2 depends on many factors, such as the shape of the construction tree, the distribution of ONUs along the tree, the price of AWGs with different numbers of ports, and the price of optical cables with different numbers of fibers.

In the following, we consider two kinds of construction tree shapes to investigate the performance of recursive partitioning and combination.

\section{A. Case 1: Construction Tree T Restricted to a Line}

In the first case, we consider the scenario in which the construction tree is restricted to a line and ONUs are uniformly distributed along the tree, as shown in Fig. 9(a).

1) Analysis. Define $l$ as the length of the construction tree $t, m$ as the number of ONUs distributed along the line, and $a$, the density, as the number of ONUs per unit length. Then, $m=l \times a$. One scheme is to use one AWG of size $1 \times m$ with cost $p(m)$. This AWG is put in the center of the construction tree to minimize the optical cable cost, as shown in Fig. 9(b).

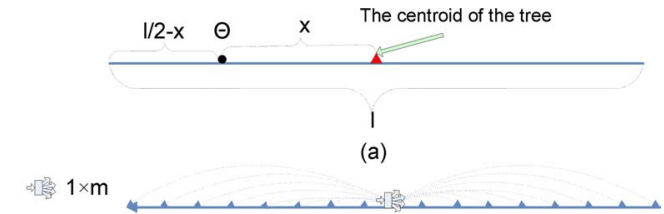

(b) The first scheme

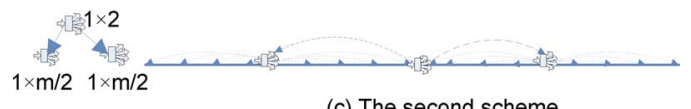

(c) The second scheme

Fig. 9. (Color online) Case 1: the construction tree is restricted to a line.

Consider the continuous scenario, at point $\theta$, which is $x$ away from the centroid of $t$; the number of optical fibers should be able to satisfy the requests from $(l / 2-x) a$ subscribers, which are further from the centroid of $t$ than $\theta$ is [see Fig. 9(a)]. The number of optical fibers at point $\theta$ is $(l / 2-x) a$. The cost of optical cables is therefore $2 \int_{0}^{l / 2} q((l / 2-x) a) \mathrm{d} x$. The total cost is then $p(m)+2 \int_{0}^{l / 2} q((l / 2-x) a) \mathrm{d} x$.

Another scheme is to use a two-stage cascaded AWG. The AWG in the first stage is of size $1 \times 2$, and the two AWGs in the second stage are of size $1 \times \mathrm{m} / 2$, as shown in Fig. 9(c). The AWG cost is $p(2)+2 p(m / 2)$. The cost of optical cables connecting one AWG of size $1 \times m / 2$ and ONUs is $2 \int_{0}^{l / 4} q(a(l / 4-x)) \mathrm{d} x$. The cost of optical cables connecting the AWG in the first stage and AWGs in the second stage is $q(1) \times l / 2$. The total optical cable cost is $4 \int_{0}^{l / 4} q(a(l / 4-x)) \mathrm{d} x+q(1) \times l / 2$. The sum of the cost of AWGs and that of optical cables is $p(2)+2 p(m / 2)+4 \int_{0}^{l / 4} q(a(l / 4-x)) \mathrm{d} x+q(1) \times l / 2$.

With $\Delta$ denoting the extra cost introduced by the second scheme as compared with the first scheme, then

$$
\begin{aligned}
\Delta= & p(2)+2 p(m / 2)-p(m)+4 \int_{0}^{l / 4} q(a(l / 4-x)) \mathrm{d} x \\
& -2 \int_{0}^{l / 2} q(a(l / 2-x)) \mathrm{d} x+q(1) l / 2 .
\end{aligned}
$$

If $\Delta>0$, the first scheme is preferred; otherwise, the second scheme is preferred. $|\Delta|$ depends on the AWG price and optical cable price.

Assume that the AWG price with $x$ ports is $c_{1} \times x^{r_{1}}$, where $0 \leqslant r_{1} \leqslant 1$. It can be easily verified that the price conforms with constraints (4) and (5). Regarding the price of optical cables, we assume the unit price of optical cable with $x$ fibers is $c_{2} \times x^{r_{2}}$, where $0 \leqslant r_{2} \leqslant 1$. If $r_{2}=0$, the optical cable cost is the same regardless of the number of contained optical fibers; if $r_{2}=1$, the optical cable cost is proportional to the number of contained optical fibers. We prove that recursive partitioning can achieve the optimal distribution AWG size in this specific case. 
Theorem 1. The proposed recursive partitioning can obtain the optimal distribution AWG size under the condition that 1) the construction tree forms a line, 2) ONUs are uniformly distributed along the line, 3) the price of an AWG with $x$ ports is $c_{1} x^{r_{1}}\left(0 \leqslant r_{1} \leqslant 1\right)$, and 4) the price of optical cable with $x$ fibers is $c_{2} x^{r_{2}}$ $\left(0 \leqslant r_{2} \leqslant 1\right)$.

Proof: Denote tree $t$ as the tree associated with a distribution AWG. We prove that if replacing this distribution AWG by two distribution AWGs and partitioning tree $t$ into two subtrees does not decrease the total cost, then any other partition will not yield a smaller cost. In this case, recursive partitioning will not miss any partition that can yield a smaller cost. For tree $t$ with length $l$ and $a \times l$ ONUs, employing one AWG with size $1 \times a l$ yields the total cost of $c_{1}(a l)^{r_{1}}$ $+c_{2} 2^{-r_{2}} a^{r_{2}} l^{r_{2}+1} /\left(r_{2}+1\right)$. Partitioning the tree into two subtrees yields the total cost of $c_{1} 2^{r_{1}}+c_{1}(a l)^{r_{1}} 2^{1-r}$ $+c_{2} l / 2+c_{2} 4^{-r_{2}} a^{r_{2}} l^{r_{2}+1} /\left(r_{2}+1\right) . \quad \Delta \quad$ equals $c_{1} 2^{r_{1}}$ $+c_{1} a^{r_{1}}\left(2^{1-r}-1\right) l^{r_{1}}+c_{2} l / 2-c_{2}\left(2^{-r_{2}}-4^{-r_{2}}\right) a^{r_{2}} l^{r_{2}+1} /\left(r_{2}+1\right)$. Considering $\Delta$ a function of the length $l$, we prove that $\Delta(l / 2)$ must be greater than zero if $\Delta(l)>0$ :

$$
\begin{aligned}
& \Delta(l / 2)=c_{1} 2^{r_{1}}+c_{1} a^{r_{1}}\left(2^{1-r}-1\right) l^{r_{1} / 2^{r_{1}}+c_{2} l / 4} \\
& -\left(2^{-r_{2}}-4^{-r_{2}}\right) c_{2} a^{r_{2}} l^{r_{2}+1} /\left[\left(r_{2}+1\right) 2^{r_{2}+1}\right] \\
& =\frac{1}{2^{r_{2}+1}}\left[c_{1} 2^{r_{1}+r_{2}+1}+c_{1} a^{r_{1}}\left(2^{1-r}-1\right) l^{r_{1}} 2^{r_{2}+1-r_{1}}\right. \\
& \left.+c_{2} l 2^{r_{2}-1}-c_{2}\left(2^{-r_{2}}-4^{-r_{2}}\right) a^{r_{2}} l^{r_{2}+1} /\left(r_{2}+1\right)\right] \\
& >\frac{1}{2^{r_{2}+1}}\left[c_{1} 2^{r_{1}}+c_{1} a^{r_{1}}\left(2^{1-r}-1\right) l^{r_{1}}+c_{2} l 2^{-1}-\left(2^{-r_{2}}\right.\right. \\
& \left.\left.-4^{-r_{2}}\right) c_{2} a^{r_{2}} l^{r_{2}+1} /\left(r_{2}+1\right)\right]=1 / 2^{r_{2}+1} \Delta(l)=0 .
\end{aligned}
$$

$\Delta(l / 2)$ is the additional cost introduced by further partitioning a subtree of tree $t . \Delta(l / 2)>0$ implies that further partitioning will not yield a smaller cost. We thereby prove that recursive partitioning can obtain the optimal distribution AWG.

2) Simulations. In the simulations, we show the impact of the number of ONUs, the AWG price, and the optical cable price on the total cost. We assume the length of the construction line equals $40 \mathrm{~km}$, and the OLT is located at the center of the construction line.

Figure 10 shows the total cost of AWGs and optical cables with different numbers of ONUs. The unit price of an AWG with $x$ ports equals $800 x^{0.4}$ dollars. The price of the optical cable with $x$ fibers equals $1000 x^{0.7}$ dollars per kilometer.

Figure 10 illustrates three kinds of costs: the cost before partitioning, the cost after partitioning, and the cost after combination. Before recursive partition-

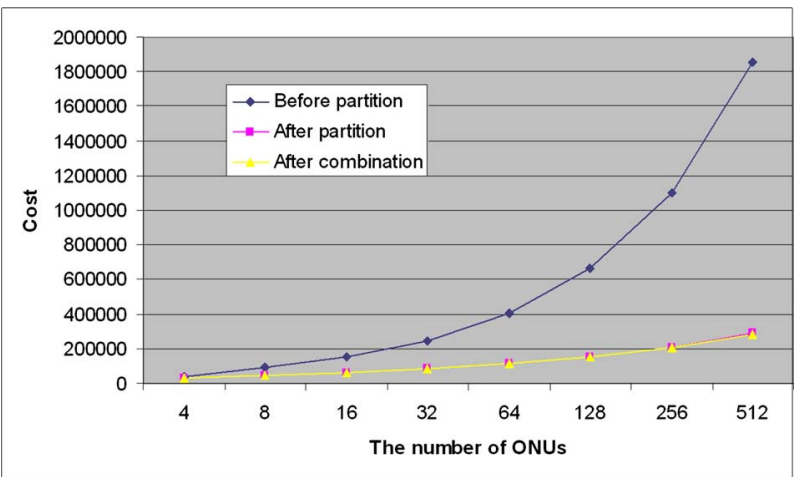

Fig. 10. (Color online) Total cost versus the number of ONUs; simulation conditions: $p(x)=800 x^{0.4}, q(x)=1000 x^{0.7}$.

ing, we use one single AWG to demultiplex wavelengths to all ONUs. For each ONU, one individual bidirectional fiber is required to connect it with the OLT. The high fiber cost results in a large total cost. After partitioning, the single large AWG is replaced by multistage cascaded AWGs. Rather than using an individual optical fiber to connect each ONU with the OLT, multiple ONUs can share one fiber. The sharing will reduce the optical cable cost, thereby resulting in the reduction of the total cost. It is shown that the total cost drops dramatically after partitioning when the number of ONUs is greater than eight. The total cost reduction with a large number of ONUs is more significant than that with a small number of ONUs. This implies that partitioning more likely occurs with a large number of ONUs. This is attributed to the fact that the number of ONUs sharing one fiber will be large if the total number of ONUs is large; this saves a large quantity of individual fibers connected to the OLT and yields a large cost reduction. Recursive combination is to optimize the intermediate AWGs as well as their associated optical cable connections. It is shown that recursive combination can decrease the cost a little when the number of ONUs equals 256 and 512 . When the number of ONUs is less than 256, recursive combination does not further decrease the cost, implying that the $1 \times 2$ intermediate AWGs are not further combined under these cases. As compared with recursive partitioning, recursive combination has a much smaller effect in the process of minimizing the total cost. There are two major reasons for this phenomenon. First, the intermediate AWGs are of size $1 \times 2$. AWGs with smaller sizes have smaller costs. Combining them further will not yield much cost reduction. Second, intermediate AWGs are located at the centroid of their corresponding subtrees, which are usually disjoint. The distance between two intermediate AWGs is relatively large as compared with the distance between distribution AWGs and their associated ONUs. A large distance results in a large cost increase if combination is performed. Hence, the intermediate AWGs are less likely to be combined. 
Figure 11 shows the impact of the AWG price on the total cost. The number of ONUs is set as 512. The price of the optical cable with $x$ fibers equals $1000 x^{0.7}$ dollars per kilometer. We observe the variation of the total cost by varying $r_{1}$ from 0.1 to 0.9 with an increment of 0.1 , and $c_{1}$ from 700 to 1500 with an increment of 200. The AWG cost is high with large $r_{1}$. Hence, the total cost increases with the increase of $r_{1}$ as shown in Fig. 11. It is also shown that the total cost increases faster than the linear increase of $r_{1}$. This is because the AWG price $c_{1} x^{r_{1}}$ increases exponentially with respect to $r_{1}$. On the other hand, since the AWG price increases linearly with $c_{1}$, the total cost increases approximately linearly with $c_{1}$ as shown in Fig. 11.

Figure 12 shows the impact of the optical cable price on the total cost. The number of ONUs is set as 512 . The unit price of an AWG with $x$ fibers equals $800^{0.4}$ dollars. Again, we vary $r_{2}$ from 0.1 to 0.9 with an increment of 0.1 , and $c_{2}$ from 700 to 1500 with an increment of 200 to observe the variation of the total cost. Figure 12 shows that the total cost increases with the increase of $r_{2}$ and $c_{2}$. This is bcause the optical cable cost increases with the increase of $r_{2}$ and $c_{2}$. However, unlike the case of the AWG price, the faster than linear increase of the optical cable price with respect to $r_{2}$ does not result in a faster than linear increase of the total cost. If the tree is partitioned in the same way for scenarios with different $r_{2}$, the AWG costs are the same, and the optical cable cost is a linear combination of several exponential functions of $r_{2}$. The increase in the total cost must be at a faster than linear pace of $r_{2}$. Hence, the slower pace of increase of the total cost must be due to the encouragement of partitioning with large $r_{2}$. This can be explained as follows. Large $r_{2}$ results in a large optical cable price, which also results in the large cost decrease when partitioning is performed. The large cost reduction associated with large $r_{2}$ encourages partitioning, which further reduces the total cost.

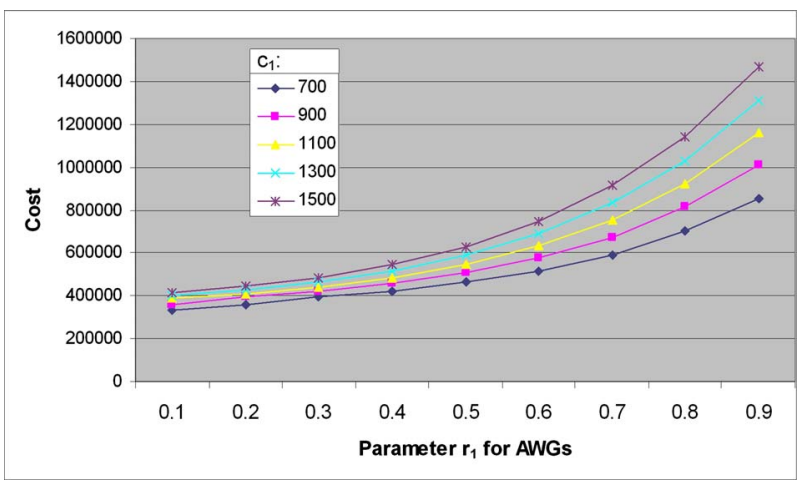

Fig. 11. (Color online) Total cost versus $r_{1}$ and $c_{1}$; simulation conditions: the construction tree forms a line, the number of ONUs is 512 , and $q(x)=1000 x^{0.7}$.

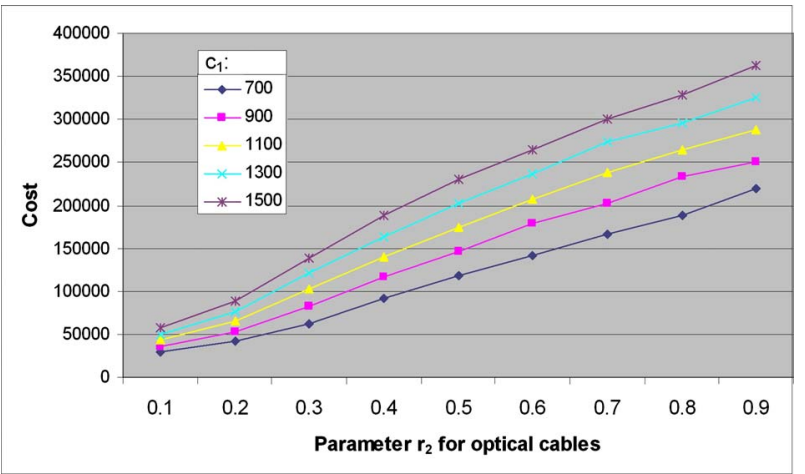

Fig. 12. (Color online) Total cost versus $r_{2}$ and $c_{2}$; simulation condition: the construction tree forms a line, the number of ONUs is 512 , and $p(x)=800 x^{0.4}$.

\section{B. Case 2: Construction Tree Forms a Binary Tree}

In the second case, we consider the scenario in which existing constructions form a binary tree as shown in Fig. 13(a). ONUs are located at leaves of the construction tree.

1) Analysis. For the first scheme of using one AWG of size $1 \times m$, this AWG is put at node $a$, which is the centroid of the construction tree, as shown in Fig. 13(a). For the second scheme of using a two-stage cascaded AWG, among which one AWG is of size $1 \times 2$ and the other two AWGs are of size $1 \times m / 2$, the AWG in the first stage is put at node $a$, and two AWGs in the second stage are put at node $b$ and node $c$, respectively. Figure 13(b) illustrates the second scheme. The difference of cable placements in the two schemes lies in edges $(a, b)$ and $(a, c)$. For the first scheme, only one fiber is needed in edge $(a, b)$ and edge $(a, c)$, respectively. For the second scheme, $m / 2$ fibers are needed in edge $(a, b)$ and edge $(a, c)$, respectively. Then

$$
\begin{aligned}
\Delta= & p(2)+2 p(m / 2)-p(m)+(q(1)-q(m / 2))(|(a, b)| \\
& +|(a, c)|) .
\end{aligned}
$$

Whether a partition is accepted depends on the AWG price, optical cable cost, and the lengths of edges.

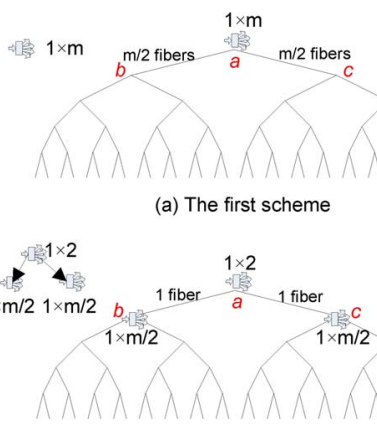

(b) The second scheme

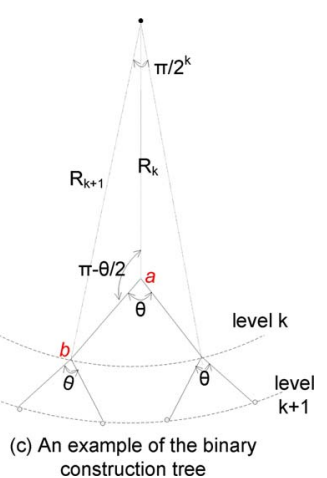

construction tree
Fig. 13. (Color online) Case 2: the construction tree $T$ forms a binary tree. 
Consider the following binary construction tree as shown in Fig. 13(c). ONUs are located at the leaves of the binary tree. Vertices at each level of the tree are uniformly distributed on a circle. For any vertex, the angle formed by its two edges connecting with its children equals $\theta$. We prove that recursive partitioning can achieve the optimal distribution AWG size for this construction tree when the AWG price $p(x)=c_{1} x^{r_{1}}(0$ $\left.\leqslant r_{1} \leqslant 1\right)$, the optical cable cost $q(x)=c_{2} x^{r_{2}}\left(0 \leqslant r_{2} \leqslant 1\right)$, and $r_{2} \geqslant r_{1}$.

Theorem 2. The proposed recursive partitioning can obtain the optimal distribution size under the condition that 1) the construction tree forms a binary tree as shown in Fig. 13(c), 2) ONUs are located at the leaves of the tree, 3) the price of an AWG with $x$ ports is $\left.c_{1} x^{r_{1}}, 4\right)$ the price of optical cable with $x$ fibers is $c_{2} x^{r_{2}}$, and 5) $r_{2} \geqslant r_{1}$.

Proof: Similar to the proof of Lemma 1, we prove that recursive partitioning can get the optimal distribution AWG by showing that, if partitioning tree $t$ into two subtrees does not decrease the total cost, then any further partitioning of tree $t$ will not yield a smaller cost. Assume that the total number of ONUs in the PON is $M$. Then, ONUs are located at a $\log _{2}^{M}$ depth of tree $t$. Each vertex at level $k$ of the tree is connected with $M / 2^{k}$ ONUs. Let $R_{k}$ be the radius of vertex at depth $k$ of the tree. Then, $R_{k+1} / \sin (\theta / 2)$ $\left.=|(a, b)| / \sin \left(\pi / 2^{k+1}\right)=R_{k} / \sin \left(\theta / 2-\pi / 2^{k+1}\right)\right)$. Let tree $t$ be the subtree with vertex $a$ as its root, where vertex $a$ is of depth $k$. Then

$$
\begin{aligned}
\Delta= & p(2)+2 p(m / 2)-p(m)+2(q(1)-q(m / 2))|(a, b)| \\
= & p(2)+2 p\left(M / 2^{k+1}\right)-p\left(M / 2^{k}\right)+2(q(1) \\
& \left.-q\left(M / 2^{k+1}\right)\right) R_{k} \frac{\sin \left(\pi / 2^{k+1}\right)}{\sin \left(\theta / 2-\pi / 2^{k+1}\right)} \\
= & c_{1} 2^{r_{1}}+c_{1}\left(2^{1-r_{1}}-1\right)\left(M / 2^{k}\right)^{r_{1}}-c_{2}\left(\left(M / 2^{k+1}\right)^{r_{2}}-1\right) \\
& \times R_{k} \frac{\sin \left(\pi / 2^{k+1}\right)}{\sin \left(\theta / 2-\pi / 2^{k+1}\right)} .
\end{aligned}
$$

Consider $\Delta$ as a function of $k$, which is the depth of the root of tree $t$. We prove that $\Delta(k+1)$ must be greater than zero if $\Delta(k)>0 . \Delta(k+1)>0$ implies that further partitioning of the subtree of tree $t$ will not yield a smaller cost:

$$
\begin{aligned}
\Delta(k) & \geqslant 0 \Rightarrow \frac{c_{1}}{c_{2}} \\
& \geqslant \frac{\left(\left(M / 2^{k+1}\right)^{r_{2}}-1\right) R_{k} \sin \left(\pi / 2^{k+1}\right)}{\left[2^{r_{1}}+\left(2^{1-r_{1}}-1\right)\left(M / 2^{k}\right)^{r_{1}}\right] \sin \left(\theta / 2-\pi / 2^{k+1}\right)} .
\end{aligned}
$$

We next prove that

$$
\begin{aligned}
\frac{R_{k} \sin \left(\pi / 2^{k+1}\right)}{\sin \left(\theta / 2-\pi / 2^{k+1}\right)} \geqslant \frac{R_{k+1} \sin \left(\pi / 2^{k+2}\right)}{\sin \left(\theta / 2-\pi / 2^{k+2}\right)}, \\
\frac{\left(M / 2^{k+1}\right)^{r_{2}}-1}{2^{r_{1}+\left(2^{1-r_{1}}-1\right)\left(M / 2^{k}\right)^{r_{1}}} \geqslant \frac{\left(M / 2^{k+2}\right)^{r_{2}}-1}{2^{r_{1}}+\left(2^{1-r_{1}}-1\right)\left(M / 2^{k+1}\right)^{r_{1}}}}: \\
\frac{R_{k} \sin \left(\pi / 2^{k+1}\right)}{\sin \left(\theta / 2-\pi / 2^{k+1}\right)}=\frac{R_{k+1} \sin \left(\pi / 2^{k+1}\right)}{\sin (\theta / 2)} \\
=\frac{R_{k+1} \sin \left(\pi / 2^{k+2}\right) 2 \cos \left(\pi / 2^{k+2}\right)}{\sin (\theta / 2)} \\
=\frac{R_{k+1} \sin \left(\pi / 2^{k+2}\right)}{\sin \left(\theta / 2-\pi / 2^{k+2}\right)} \\
\\
\times \frac{2 \cos \left(\pi / 2^{k+1}\right) \sin \left(\theta / 2-\pi / 2^{k+1}\right)}{\sin (\theta / 2)} \\
=\frac{R_{k+1} \sin \left(\pi / 2^{k+2}\right)}{\sin \left(\theta / 2-\pi / 2^{k+2}\right)} \\
\times \frac{\sin (\theta / 2)+\sin \left(\theta / 2-\pi / 2^{k}\right)}{\sin (\theta / 2)} \\
\times \frac{R_{k+1} \sin \left(\pi / 2^{k+2}\right)}{\sin \left(\theta / 2-\pi / 2^{k+2}\right)} .
\end{aligned}
$$

Since $r_{2} \geqslant r_{1}, \quad\left(2^{1-r_{1}}-1\right)\left(M / 2^{k}\right)^{r_{1}+r_{2}} 2^{-r_{2}}\left(2^{-r_{1}}-2^{-r_{2}}\right) \geqslant 0$. Then, we have

$$
\begin{aligned}
\left(M / 2^{k}\right)^{r_{2}} 2^{r_{1}-r_{2}}+\left(2^{1-r_{1}}-1\right)\left(M / 2^{k+1}\right)^{r_{1}+r_{2}}-\left(2^{1-r_{1}}-1\right) \\
\quad \times\left(M / 2^{k}\right)^{r_{1}}>\left(M / 2^{k}\right)^{r_{2}} 2^{r_{1}-2 r_{2}}+\left(2^{1-r_{1}}-1\right) \\
\quad \times\left(M / 2^{k}\right)^{r_{1}+r_{2}} 2^{-2 r_{2}}-\left(2^{1-r_{1}}-1\right) \\
\quad \times\left(M / 2^{k+1}\right)^{r_{1}} \Rightarrow \frac{\left(M / 2^{k+1}\right)^{r_{2}}-1}{2^{r_{1}+\left(2^{1-r_{1}}-1\right)\left(M / 2^{k}\right)^{r_{1}}}} \\
>\frac{\left(M / 2^{k+2}\right)^{r_{2}}-1}{2^{r_{1}}+\left(2^{1-r_{1}}-1\right)\left(M / 2^{k+1}\right)^{r_{1}}}
\end{aligned}
$$

Therefore,

$$
\begin{aligned}
& \frac{R_{k+1} \sin \left(\pi / 2^{k+2}\right)}{\sin \left(\theta / 2-\pi / 2^{k+2}\right)} \frac{\left(M / 2^{k+2}\right)^{r_{2}}-1}{2^{r_{1}}+\left(2^{1-r_{1}}-1\right)\left(M / 2^{k+1}\right)^{r_{1}}} \\
& <\frac{R_{k} \sin \left(\pi / 2^{k+1}\right)}{\sin \left(\theta / 2-\pi / 2^{k+1}\right)} \frac{\left(M / 2^{k+1}\right)^{r_{2}}-1}{2^{r_{1}}+\left(2^{1-r_{1}}-1\right)\left(M / 2^{k}\right)^{r_{1}}} \\
& \leqslant \frac{c_{1}}{c_{2}} \Rightarrow \Delta(k+1)>0 .
\end{aligned}
$$

$\Delta(k+1)$ is the additional cost introduced by further partitioning a subtree of tree $t . \Delta(k+1)>0$ implies that further partitioning will not yield a smaller cost. We thereby prove that recursive partitioning can ob- 
tain the optimal distribution AWG.

2) Simulations. In the simulations, we set the distance between the OLT and the ONUs as $20 \mathrm{~km}$, the number of ONUs as 512 , and $\theta$ as $120^{\circ}$.

Figures 14 and 15 show the impact of the AWG price and optical cable price on the total cost, respectively. In Fig. 14, $q(x)=1000 x^{0.7}$. In Fig. 15, $p(x)=800^{0.4}$. Both $r_{1}$ in Fig. 14 and $r_{2}$ in Fig. 15 are varied from 0.1 to 0.9. Both $c_{1}$ in Fig. 14 and $c_{2}$ in Fig. 15 are varied from 700 to 1500 . Figures 14 and 15 show that the total cost increases with the increase of $r_{1}, r_{2}, c_{1}$, and $c_{2}$. As compared with those shown in Fig. 11 and Fig. 12, the pace of increase of the total cost with respect to $r_{1}$ and $r_{2}$ are reduced, respectively. This is attributed to the fact that the sizes of distribution AWGs employed in this scenario are generally larger than the sizes of AWGs employed in the scenario in which the construction tree forms a line. Comparing Eqs. (11) and (13), we can find that the decrease of the optical cable cost in the case with a binary construction tree is slower than that in the case where the construction tree forms a line. The small decrease of the optical cable cost discourages partitioning in the case of a binary construction tree. Hence, large sizes of distribution AWGs are yielded. Since $r_{1}<1, c_{1} x^{r_{1}}$ increases slower with larger $x$. We therefore get a slower pace of increase of the total cost.

\section{CONCLUSION}

We have presented a scheme to optimize the tradeoff between the AWG cost and the optical cable cost of deploying ODNs of WDM PONs. Specifically, we decompose the problem into two subproblems: minimize the optical cable cost for a given cascaded AWG structure, and then decide the cascaded AWG structure that minimizes the total cost of AWGs and optical cables. The former problem is further divided into two subproblems: decide ONUs connected to each AWG, and decide geometric positions of AWGs. We have dis-

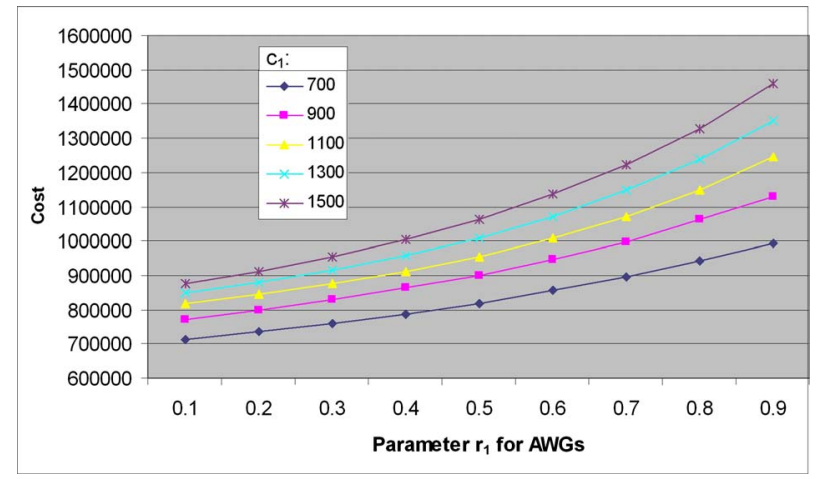

Fig. 14. (Color online) Total cost versus $r_{1}$ and $c_{1}$; simulation condition: the construction tree is a binary tree, the number of ONUs is 512 , and $q(x)=1000 x^{0.7}$.

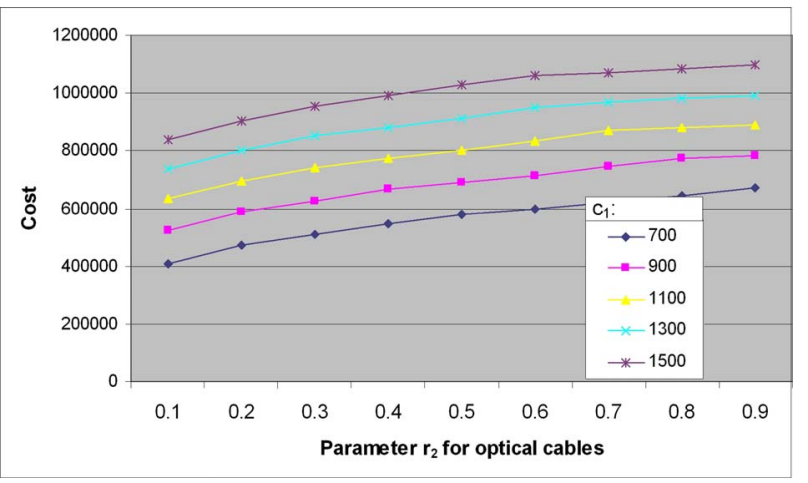

Fig. 15. (Color online) Total cost versus $r_{2}$ and $c_{2}$; simulation condition: the construction tree is a binary tree, the number of ONUs is 512 , and $p(x)=800 x^{0.4}$.

cussed these problems in detail and proposed corresponding algorithms to solve them. We have also analyzed the impact of the fiber cost, AWG cost, and subscriber density by using our proposed algorithms for deploying an ODN of a WDM PON. The WDM PON has to overcome a few hurdles to see its widespread deployment. The contributions of this work are bridging the gap toward this promising solution for an access technology with high-bandwidth provisioning.

\section{ACKNOWLEDGMENT}

This work was supported in part by the National Science Foundation (NSF) under grant 0726549.

\section{REFERENCES}

[1] A. Banerjee, Y. Park, F. Clarke, H. Song, S. Yang, G. Kramer, K. Kim, and B. Mukherjee, "Wavelength-division-multiplexed passive optical network (WDM-PON) technologies for broadband access: a review," J. Opt. Netw., vol. 4, no. 11, pp. 737758, Nov. 2005.

[2] F. Effenberger, D. Clearly, O. Haran, G. Kramer, R. D. Li, M. Oron, and T. Pfeiffer, "An introduction to PON technologies [topics in optical communications]," IEEE Commun. Mag., vol. 45, no. 3, pp. S17-S25, March 2007.

[3] C.-H. Lee, S.-M. Lee, K.-M. Choi, J.-H. Moon, S.-G. Mun, K.-T. Jeong, J. H. Kim, and B. Kim, "WDM-PON experiences in Korea," J. Opt. Netw., vol. 6, no. 5, pp. 451-464, 2007.

[4] K. Okamoto, Fundamentals of Optical Waveguides. New York, NY: Academic, 2006.

[5] B. Kuhlow, G. Przyrembel, E. Pawlowski, M. Ferstl, and W. Furst, "AWG-based device for a WDM overlay PON in the $1.5 \mathrm{~m}$ band," IEEE Photon. Technol. Lett., vol. 11, no. 2, pp. 218-220, Feb. 1999.

[6] G. Maier, M. Martinelli, A. Pattavina, and E. Salvadori, "Design and cost performance of the multistage WDM-PON access networks," J. Lightwave Technol., vol. 18, no. 2, pp. 125-143, Feb. 2000.

[7] I. Tsalamanis, M. Toycan, and S. Walker, "Study of hybrid cascaded AWG-based access network topology," in 2006 Int. Conf. on Transparent Optical Networks, Nottingham, UK, June 1822, 2006, vol. 4, pp. 76-79.

[8] I. Tsalamanis, E. Rochat, S. Walker, M. Parker, and D. Holburn, "Experimental demonstration of cascaded AWG access network featuring bi-directional transmission and polarization 
multiplexing," Opt. Express, vol. 12, no. 5, pp. 764-769, 2004.

[9] F. I. El-Nahal and R. J. Mears, "Multistage WDM access architecture employing cascaded AWGs," Opt. Fiber Technol., vol. 15, pp. 181-186, March 2009.

[10] S.-M. Lee, S.-G. Mun, M.-H. Kim, and C.-H. Lee, "Demonstration of a long-reach DWDM-PON for consolidation of metro and access networks," J. Lightwave Technol., vol. 25, no. 1, pp. 271-276, Jan. 2007.

[11] M. Hajduczenia, B. Lakic, H. J. da Silva, and P. P. Monteiro, "Optimized passive optical network deployment," J. Opt. Netw., vol. 6, no. 9, pp. 1079-1104, Sept. 2007.

[12] J. Li and G. Shen, "Cost minimization planning for passive optical networks," in Nat. Fiber Optic Engineers Conf., San Diego, CA, OSA Technical Digest (CD), Washington, DC: Optical Society of America, Feb. 24, 2008, paper NThD1.

[13] S. Khan, "Heuristics-based PON deployment," IEEE Commun. Lett., vol. 9, no. 9, pp. 847-849, Sept. 2005.

[14] F. K. Hwang and D. S. Richards, "Steiner tree problems," Networks, vol. 22, no. 1, pp. 55-89, 1992

[15] S. Gokhale, "Deployment of fiber optic networks through underground sewers in North America," J. Transp. Eng., vol. 132, no. 8, pp. 672-682, 2006.

[16] J. K. Jeyapalan, "Using existing infrastructure to speed FTTH deployment," Broadband Properties Mag., vol. 2007, no. 3, pp. 61-70, March 2007.

[17] M. Garey and D. Johnson, Computers and Intractability: a Guide to the Theory of NP-Completeness. New York, NY: W. H. Freeman, 1979.

[18] D. S. Johnson and K. A. Niemi, "On knapsacks, partitions, and a new dynamic programming technique for trees," Math. Op. Res., vol. 8, no. 1, pp. 1-14, 1983.

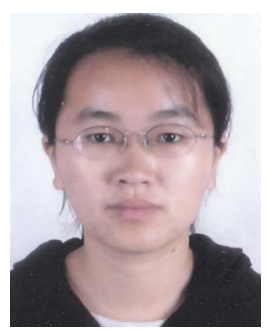

Jingjing Zhang received a B.E. degree from Xi'an Institute of Posts and Telecommunications, China, in 2003, and an M.E. degree from Shanghai Jiao Tong University, China, in 2006, both in electrical engineering. She is working toward her $\mathrm{Ph}$.D. degree in electrical engineering at New Jersey Institute of Technology, Newark. Her research interests include planning, capacity analysis, and resource allocation of broadband access networks, and quality of experience provisioning in next-generation networks.

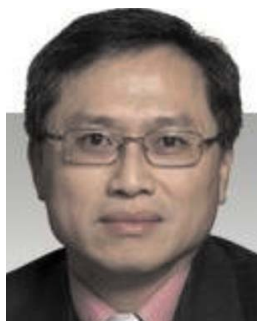

Nirwan Ansari received the B.S.E.E. (summa cum laude) with a GPA of 4.00/4.00) from the New Jersey Institute of Technology (NJIT), Newark, in 1982, the M.S.E.E. degree from the University of Michigan, Ann Arbor, in 1983, and the $\mathrm{Ph} . \mathrm{D}$. degree from Purdue University, West Lafayette, Indiana, in 1988. He joined NJIT's Department of Electrical and Computer Engineering as an Assistant Professor in 1988 and has been a Full Professor since 1997. He has also assumed various administrative positions at NJIT. He authored Computational Intelligence for Optimization (Springer, 1997, translated into Chinese in 2000) with E. S. H. Hou and edited Neural Networks in Telecommunications (Springer, 1994) with B. Yuhas. His current research focuses on various aspects of broadband networks and multimedia communications. Among around 30 patent applications he has contributed, three have been issued and others are pending. He has also contributed more than 300 technical papers, over one third of which were published in widely cited refereed journals or magazines. He is a Senior Technical Editor of the IEEE Communications Magazine and also serves on the Advisory Board and Editorial Board of five other journals. He has been serving the IEEE in various capacities such as Chair of the IEEE North Jersey COMSOC Chapter, Chair of the IEEE North Jersey Section, Member of the IEEE Region 1 Board of Governors, Chair of the IEEE COMSOC Networking TC Cluster, Chair of the IEEE COMSOC Technical Committee on Ad Hoc and Sensor Networks, and Chair/TPC Chair of several conferences/ symposia. Some of his recent awards and recognitions include an IEEE Fellow (Communications Society), IEEE Leadership Award (2007, from Central Jersey/Princeton Section), the NJIT Excellence in Teaching in Outstanding Professional Development (2008), IEEE MGA Leadership Award (2008), the NCE Excellence in Teaching Award (2009), and designation as an IEEE Communications Society Distinguished Lecturer. 